\title{
Anemia Tardía en Niños con Incompatibilidad Rh y ABO.
}

\author{
Dres. : Manuel Olivares $\mathrm{G}^{1}{ }^{1}$; Liliana Schlesinger ${ }^{1}{ }^{1}$; Ernesto Rios L. ${ }^{2}$ \\ Inés Lopez T.; Abraham Stekel G.'. \\ Late Anemia in Infants With $\mathrm{Rh}$ \\ ABO Incompatibilities
}

The spontaneous evolution of the hematocrit, hemoglobin and reticulocyte count values of 131 term infants with clinical and laboratory diagnosis of Rh or ABO incompatibility was studied. They vere classified as follows: 17 infants with $R$ h incompatibility not subject to exchange tfansfusion, 36 infants with Rh incompatibility requiring one exchange transfusion, 17 infants with $\mathrm{Rh}$ incompatibility requiring two or more exchange transfusions, 15 infants with $A B O$ incompatibility who dis not require exchange transfusion and 46 infants with $A B O$ incornpatibility who did not require exchange transfusion. Thirty five normal infants served as controls. Hematological values were determined at $15,30,45,60,90,180$ and 270 days of age. Signilicant differences were present at 15,30 and 60 days of age between normals and patients with incompatibility. Despite the disadvantage in hematocrit and hemoglobin concentration experienced at birth and up to three month of age by infants with incompatibility, differences disappear between 90 and $\mathbf{1 8 0}$ days of age. This is important in planning the treatment of the exagerated physiologic anemia of these patients.

Un número significativo de los niños con enfermedad hemolítica del recién nacido, desarrollan en las primeras semanas de vida, lo que se ha denominado "anemia tardia"] - $2 \cdot 3 \cdot 4$, atribuida a la persistencia de la hemólisis producida por los anticuerpos específicos adquiridos por vía transplacentaria ${ }^{\mathbf{3}}$.

1 Instituto de Nutrición y Tecnología de los Alimentos, Universidad de Chile.

2 Facultad de Medicina, División Oriente, Universidad de Chile.
En la terapia de esta anemia se utiliza a menudo la transfusión sanguínea, sometiendo al niño a los riesgos inherentes a este procedimiento y a una eventual frenación de la eritropoyesis ${ }^{5-6}$.

El objetivo del presente trabajo fue estudiar la evolución espontánea de la anemia tardía, en niños con incompatibilidad, Rh y de grupo clásico.

\section{MATERIAL Y METOBO}

Durante los años 1972 a 1976 , en la Unidad de Hematología del ex Hospital Manuel Arriarán, 
se siguió longitudinalmente un grupo de 131 nifios con antecedentes de incompatibilidad Rh o de grupo clásico, los que se compararon con 35 lactantes sin incompatibilidad (Tabla 1).

\section{Tabla $]$.}

Composición de los Grupos

\section{Pacientes}

No Casos

Iacompatibilidad $R$ h sin exsangu íneo-transfusión

Incompatibilidad Rli con

I exsanguineo-transfusión

Incompatibilidad Rh con 2 o más exsanguineo .transfusiones

Incompatibilidad ABO sin exsanguíneo transfusión

Incompatibilidad ABO con

1 exsanguíneo-transfusión

Controles

Todos los lactantes habían nacido en la Maternidad del ex Hospital San Borja, de parto de término con un peso de nacimiento superior a $2.500 \mathrm{~g}$.

Para el diagnóstico de incompatibilidad $R h$ se exigió: madre $R h(-)$, niffo $R h(+)$ y prueba de Coombs directa del cordón (+). Para el diagnóstico de incompatibilidad de grupo clásico se exígió: madre 0 y niño $A$ ó $B$, asociado a ictericia de comienzo precoz (menos de 24 horas) y/o hiperbilirrubinemia (bilirrubina total mayor de 15 $\mathrm{mg} / \mathrm{d} 1$ ). En 53 de los niños con incompatibilidad
Rh y en 46 de los con incompatibilidad ABO fue necesario realizar una o más exsanguíneotransfusiones, las que se indicaron y realizaron de acuerdo a los criterios clásicos para este procedimiento ${ }^{1}$.

A $\operatorname{los} 15,30,45,60,90,180$ y 270 días de vida se realizó en control antropométrico y se obtuvo por punción capilar una muestra sangui. nea, realizándose las siguientes determinaciones: hematocrito (microhematocrito), recuento de reticulocitos. En un número menor de casos se determinó la concentración de hemoglobina (cianmetahemoglobina).

Todos los niños recibieron similares indicaciones y no se prescribieron hematínicos, excepto hierro a aquellos pacientes que presentaron una anemia hipocroma a los 180 días, excluyéndoseles del análisis a los 270 dias.

\section{RESULTADOS}

El hematocrito en los nifros controles (Ta. bla 2) experimentó el clásico descenso postnatal, alcanzando su valor más bajo a los 90 dias de vida. A partir de este momento se produjo un incremento gradual del hematocrito, alcanzando los valores propios del lactante mayor. Los pacientes con incompatibilidad $\mathrm{Rh}$ presentaron a los 15 dias hematocritos menores, permaneciendo este examen significativamente más bajo hasta los 60 dias, para de ahí en adelante alcanzar las cifras de los contrules. A los 60 días el grupo con dos exsanguineo-transfusiones tuvo hematocritos significativamente más bajos que el de los grupos sin recambio o con una exsanguíneotransfusión.

Tabla 2.

Evolución Espontánea del Hematocrito (\%) en Ninos con Incompatibilidad Rh*.

\begin{tabular}{|c|c|c|c|c|c|c|c|}
\hline Dias & 15 & 30 & 45 & 60 & 90 & 180 & 270 \\
\hline $\begin{array}{l}\text { Incompdtibilidad RI: sin } \\
\text { exsanguíneo-transfusion }\end{array}$ & $\begin{array}{c}33.5 \pm 8.4^{* *} \\
(12)\end{array}$ & $\begin{array}{c}28.0 \pm 5.0^{n *} \\
.151\end{array}$ & $\begin{array}{c}29.9 \pm 6.2^{* *} \\
\text { (8) }\end{array}$ & $\begin{array}{c}32.0 \pm 2.0^{* *} \\
(12)\end{array}$ & $\begin{array}{l}34.2 \pm 2.9 \\
\quad(13)\end{array}$ & $\begin{array}{c}35.7 \pm 4.1 \\
(10)\end{array}$ & $\begin{array}{c}37.1 \pm 2.5 \\
(8)\end{array}$ \\
\hline $\begin{array}{l}\text { Incompatibllidad Rh con } \\
1 \text { cxsanguíteo Iranstusión }\end{array}$ & $\begin{array}{l}35.5 \pm 6.3 * * \\
1181\end{array}$ & $\begin{array}{c}28.9 \pm 4.8^{* *} \\
(31)\end{array}$ & $\begin{array}{c}26.9 \pm 2.8^{* *} \\
(131)\end{array}$ & $\begin{array}{c}30.0 \pm 3.0 * * \\
(27)\end{array}$ & $\begin{array}{c}33.0 \pm 3.5 \\
(23)\end{array}$ & $\begin{array}{l}36.6 \pm 2.3 \\
(18)\end{array}$ & $\begin{array}{c}37.3 \pm 3.0 \\
(1 \leq \mathbf{3}\end{array}$ \\
\hline $\begin{array}{l}\text { Incompatibilidat Rh con } 2 \\
\text { exsanguínco- lfansfusiones }\end{array}$ & $\begin{array}{c}35.6 \pm 6.0^{* *} \\
(8)\end{array}$ & $\begin{array}{c}29.0 \pm 6.8 * \\
(14)\end{array}$ & $\begin{array}{c}25.6 \pm 3.4^{* *} \\
\text { (13) }\end{array}$ & $\begin{array}{c}27.6 \pm 3.1^{* *} \\
(14)\end{array}$ & $\begin{array}{c}32.0 \pm 2.2^{* *} \\
(13)\end{array}$ & $\begin{array}{l}36.5 \pm 2.0 \\
(10)\end{array}$ & $\begin{array}{c}37.0 \pm 2.5 \\
(10)\end{array}$ \\
\hline Contrales & $\begin{array}{c}57.5 \pm 7.9 \\
130)\end{array}$ & $\begin{array}{c}46.5 \pm 7.2 \\
(27)\end{array}$ & $\begin{array}{c}39.4 \pm 3.5 \\
(23)\end{array}$ & $\begin{array}{l}34.5 \pm 3.5 \\
(21)\end{array}$ & $\begin{array}{l}33.5 \pm 1.8 \\
(17)\end{array}$ & $\begin{array}{l}36.4 \pm 3.4 \\
\text { (11) }\end{array}$ & $\begin{array}{l}35.1 \pm 2.0 \\
(9)\end{array}$ \\
\hline
\end{tabular}

* Promedio \pm 1 D.S. Número de casos en paréntesis

* Diferencia significativa con respecto al control.

En los sujetos con incompatibilidad ABO (Ta. bla 3) la disminución postratal del hematocrito fue menos pronunciada; a pesar de ello los valores fueron significativamente más bajos que en el grupo control, pero sólo hasta los 45 dias en los niños no recambiados y hasta los 60 días en los que recibieron una exsanguineo-transfusión. 
Tabla 3.

Evolución Espontánea del.Henatocuito (\%) en Niños con Incompatibilidad ABO*

\begin{tabular}{|c|c|c|c|c|c|c|c|}
\hline Dias & 15 & 30 & 45 & 60 & 90 & $1 \mathrm{BO}$ & 230 \\
\hline 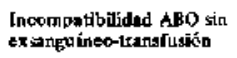 & $\begin{array}{c}44.1 \pm 8.1 \cdots \\
(8)\end{array}$ & $\begin{array}{c}33.6 \pm 6.0^{* *} \\
(1 \mathrm{l})\end{array}$ & $\begin{array}{l}31.9 \pm 4.7 * * \\
\text { (B) }\end{array}$ & $\begin{array}{c}32.9 \pm 5.2 \\
(11)\end{array}$ & $\begin{array}{c}34.0 \pm 3.5 \\
1123\end{array}$ & $\begin{array}{c}31.1 \pm 2.9 \\
(9]\end{array}$ & $3 \leq-5 \pm 1.5$ \\
\hline 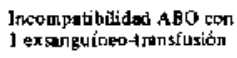 & $\begin{array}{c}42.4 \pm 71^{n "} \\
(22)\end{array}$ & $\begin{array}{c}3-6 \pm 5.3 \mathrm{me} \\
(34)\end{array}$ & $\begin{array}{c}31.1 \pm 4,849 \\
(31)\end{array}$ & $\begin{array}{c}30.4 \pm 3.577 \\
(27)\end{array}$ & $\begin{array}{c}32.9 \pm 3.0 \\
(33)\end{array}$ & $\begin{array}{l}35.7 \pm 2.1 \\
(30)\end{array}$ & $\begin{array}{l}36.2 \pm 2.3 \\
\quad(22)\end{array}$ \\
\hline Controles & $\begin{array}{c}57.5 \pm 7.9 \\
\text { (30) }\end{array}$ & $\begin{array}{c}465 \pm 7.2 \\
(27)\end{array}$ & $\begin{array}{c}39.4 \pm 3.5 \\
(23)\end{array}$ & $\begin{array}{c}34.5 \pm 3.5 \\
(21)\end{array}$ & $\begin{array}{c}33.5 \pm 1.8 \\
(17)\end{array}$ & $\begin{array}{l}36.4 \pm 3.4 \\
\text { (1I) }\end{array}$ & $\begin{array}{c}35.1 \pm 2.1 \\
\text { (9) }\end{array}$ \\
\hline
\end{tabular}

* Promedio \pm 1 D.S. Número de casos en paréntesis.

** Diferencia significativa respecto del control.

La concentración de hemoglobina sufrió cambios similares a los del hematocrito en los lactantes controles (Figura 1) descendiendo rápidamente durante los primeros 60 días de vida llegando a su valor más bajo a los 90 días, para con posterioridad aumentar su concentración. En los niffos con incompatibitidad $R$ h la velocidad del descenso entre los 15 a 30 días fue casi igual a la del grupo control, pero llegaron a cifras de hemoglobina muy por debajo de las del grupo normal, observándose la menor concentración de hemoglobina a los 45 días de vida. En los sujetos con incompatibilidad $A B O$ (Figura 2) el descenso de la hemoglobina fue menos marcado, llegando al valor más bajo a los 60 días en los niños con una exsanguíneo-transfusión.

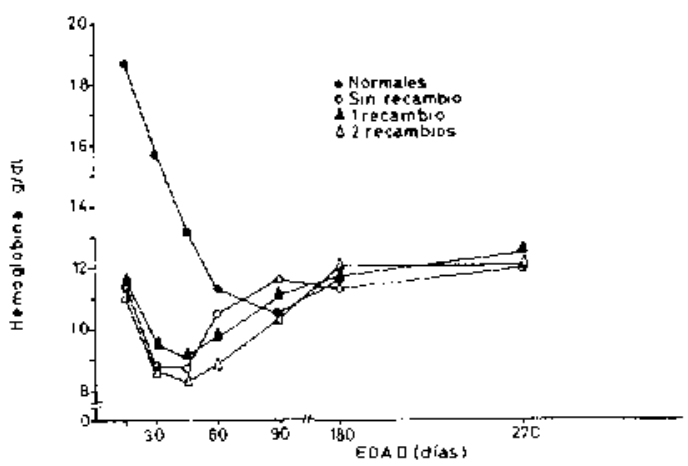

Figura 1. Evolución de la concentración de hemoglobina en nitios con incompatibilidad Rh.

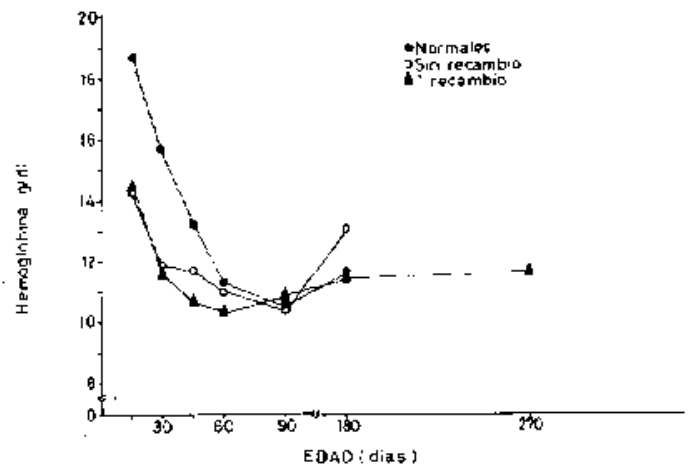

Figura 2. Evolución de la concentración de hemoglobina en nî̃ios con incompatibilidad ABO.
E] recuento de reticulocitos en los nir̃os controles (Figura 3) permaneció bajo en los primeros 30 días de vida, para aumentar a partir de los 45 dias, alcanzando su mayor valor a los 90 días, edad que coincidió con la concentración menor de hemoglobina. En los sujetos con incompatibilidad $\mathrm{Rh}$, el recuento de reticulocitos aumentó más precozmente y llegó a valores bastante más altos que los del grupo control. Los niños con incompatibilidad $\mathrm{ABO}$ (Figura 4) también mostraron un incremento precoz del recuento de reticulocitos; sin embargo este aumento es de menor cuatía que el del grupo con incompatibilidad Rh.

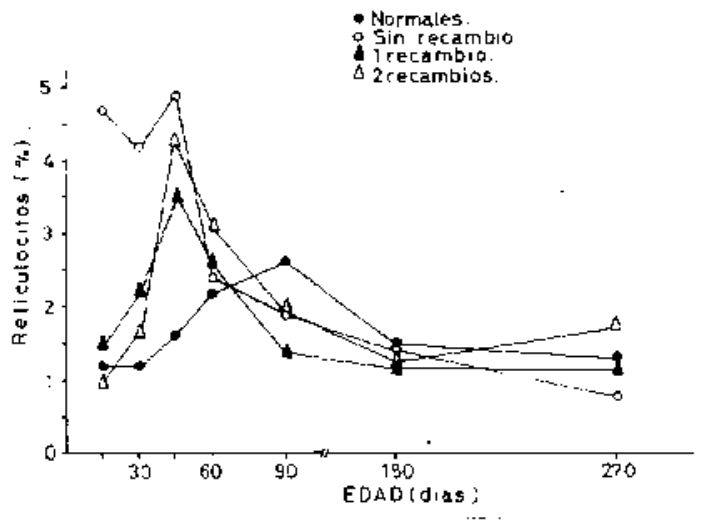

Figura 3. Evolución del porcentaje de Jecitulocitos en niños con incompatibilidad $\mathrm{Rh}$.

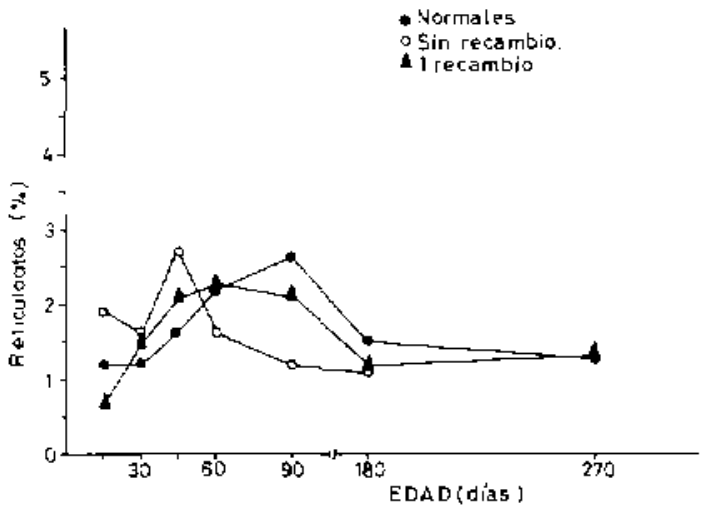

Figura 4. Evolución del porcentaje de retjeulocitos en nitos con incompatiblidad ABO. 
Los sujetos con incompatibilidad $\mathrm{Rh}$ o $\mathrm{ABO}$, presentaron un peso promedio similar al del grupo control, no encontrándose diferencias significativas a las edades estudiadas.

\section{DISCUSION}

El recién nacido presenta una alta concentración de hemoglobina, estimada a nivel del cordón en $17.6 \mathrm{~g} / \mathrm{d} \mathrm{l}^{7}$. En el período postnatal se produ. ce un gradual descenso, debido a la frenación de la eritropoyesis determinada por el aumento de la saturación de oxígeno de la hemoglobina, que ocurre una vez iniciada la respiración ${ }^{7}$. Esta caída llega a su máximo alrededor de las 6 a 8 semanas de vida, edad a a partir de la cual se produce una reactivación de la eritropoyesis, que es evidenciable por un incremento del recuento reticulocitario y un gradual aumento de la hemoglobina ${ }^{7 \cdot 8}$. El recién nacido prematuro, por razones que se ignoran, experimenta un descenso de la hemoglobina más pronunciado y $\operatorname{precoz}^{9}$.

En los lactantes con antecedentes de incompatibilidad $\mathrm{Rh} \circ \mathrm{ABO}$, se observa frecuentemente una exageración de la caída físiológica de la concentración de hemoglobina, la que alcanza su punto más bajo alrededor de las 4 a 6 semanas de vida $3 \cdot 2-3-4$. Esta anemia ocurre tanto en sujetos sometidos a una exsanguíneo-transfusión, como en pacientes no recambiados y es más frecuente en aquellos lactantes que tuvieron una hemoglobina del cordón inferior a $12 \mathrm{~g} / \mathrm{dl}^{3} 0$ una hemoglobina menor de $15 \mathrm{~g} / \mathrm{d} l$ durante la primera semana de vida ${ }^{1-2-10}$ o inferior a 14 $\mathrm{g} / \mathrm{d} 1$ post recambio ${ }^{3}$.

En nuestra casuística la mayoría de los pacientes con incompatibilidad presentó una hemoglobina bajo lo normal en el curso de los tres primeros meses de vida. Al desglosarlos según el tipo de incompatibilidad, se pudo apreciar que el $71.8 \%$ de los lactantes con incompatibilidad Rh tuvo una hemoglobina inferior a $9 \mathrm{~g} / \mathrm{dl}$, mientras que en el grupo con incompatibilidad $A B O$ esta incidencia fue bastante menor, de sólo un $23,1 \%$. Por no disponer de determinaciones durante la primera semana de vida, no nos fue posible establecer correlaciones entre la incidencia de anemia y la cifra de hemoglobina inicial.

En la génesis de esta anemia, además de las condiciones que determinan la caída postnatal fisiológica de la concentración de hemoglobina, intervienen otros mecanismos. El principal de ellos obedece al traspaso vía transplacentaria de un anticuerpo materno (IgG) capaz de destruir a los eritrocitos incompatibles del recién nacido ${ }^{1}$. Este anticuerpo es capaz de afectar tanto a los glóbulos rojos presentes en el nacimiento, como a los producidos una vez reiniciada la eritropoyesis. La vida media de esta inmunoglobulina en la circulación del recién nacido, se ha estimado en 2-3 semanas ${ }^{1-2}$. Menos frecuentemente este anticuerpo es capaz de dafiar a los precursores de la serie eritroide y existen en la literatura algunos casos documentados en los cuales también ha participado este mecanismo ${ }^{7-8}$. Se ha postulado como otro factor participante a una posible falencia de algún factor especifico para la eritropoyesis ${ }^{8}$, especialmente un déficit de ácido fóljco, dado que en los cuadros hemolíticos existen requerimientos aumentados de esta vitamina; sin embargo esta posibilidad no ha sido demostrada.

En nuestro estudio, al igual que en el de otros autores, se pudo apreciar que los niños con incompatibilidad llegan a cifras de hemoglobina bastante más bajas que los controles normales; sin que se aprecie una reanudación efectiva de la eritropoyesis ${ }^{1-2}-3-4$ pese a existir evidencias de una reiniciación más precoz đe la producción eritrocitaria a juzgar por los valores de reticulocitos. Este hecho pudiera deberse a la existencia de una hipofunción medular relativa en estos niños en la que la respuesta medular no seria suficiente para compensar la destrucción eritrocitaria, o bien debido a la existencia de un cierto grado de compromiso funcional medular, determinado por la acción de los anticuerpos maternos o menos posiblemente debido a la carencia de algún factor específico para la eritropoyesis.

De acuerdo a nuestras observaciones, en los niños con incompatibilidad Rh o ABO la mayor destrucción de los eritrocitos ocurriría en el curso de las dos primeras semanas de vida, siendo con posterioridad la velocidad de caida de la hemoglobina bastante similar a la de los niños normales.

En nuestros casos se pudo apreciar que si bien los niños con incompatibilidad llegan a yalores más bajos de hemoglobina en los primeros dos meses de vida, esta anemia se recupera espontáneamente a los 3 meses, sin que sea necesario la realización de una transfusión sanguínea.

Las curvas de peso de nuestros pacientes con incompatibilidad no fueron diferentes de las observadas en los sujetos controles, pareciendo no existir ninguna desventaja importante en el crecimiento, asociado a niveles de hemoglobina subnormales durante un período de algunas semanas.

Creemos que desde el punto de vista hematológico, no se justifica ningún tipo de tratamiento para la anemia tardía, siendo aconsejable el control periódico de la concentración de hemoglobina durante los tres primeros meses de vida y sólo excepcionalmente en casos muy calificados podría estar indicada una transfusión de glóbulos 
rojos concentrados como tratamiento de esta anemia. Este control periođico debe ser más frecuente en los niños con incompatibilidad $\mathrm{Rh}$, en los cuales lá caída de la hemoglobina es más pronunciada y la frecuencia de anemia es mayor.

\section{RESUMEN}

Se estudió la evolución espontánea del hema. tocrito, hemoglobina y reticulocitos en 131 niños nacidos de término con antecedentes clínicos y de laboratorio de incompatibilidad $\mathrm{Rh}$ o $\mathrm{ABO}$. Estos se subdividieron en los siguientes grupos: Diecisiete niños con incompatibilidad Rh que no se sometieron a exsanguíneo-transfusión. Treinta $y$ seis niños con incompatibilidad $R h$ con una exsanguíneo-transfusión. Diecisiete niños con incompatibilidad $\mathrm{Rh}$ con dos o más exsanguíneo-transfusiones. Quince niños con incompatibilidad ABO sin exsanguíneo-transfusión. Cuarenta y seis niños con incompatibilidad $\mathrm{ABO}$ con una exsanguíneo-transfusión. Treinta y cinco njî̃os normales constituyeron el grupo control. La evolución de los valores hematológicos se estudió a los 15, 30, 45, 60, 90,180 y 270 días. Entre los 15 y los 60 días de vida se observaron cifras de hematocrito $y$ hemoglobina significativamente menores en los pacientes con incompatibilidad. En controles posteriores los grupos con incompatibilidad y los normales evolucionaron en forma similar no existiendo diferencias significativas después de los 90 días đe vida. A pesar de la desventaja inicial de los niños con incompatibilidad, esto no influye en los valores hematológicos de $\operatorname{los} 90$ a 180 días de edad. Esto tendría importancia èn el tratamiento de la anemia fisiológica exagerada de estos niños.

\section{REFERENCIAS}

1 Mollison, P.L.: Blood transtusion in clinical mitdicine. Fifth edition. Blackwell Scientific Publications, Oxford 1974.

2 Fraser, I.D.; Oppé, T.E.; Tovey, G.H.; Webb, D.A.: Post-cxchange anacmia in $R h$ haemolytic disease. Lancet II: $1309,1964$.

3 Hurdie, A.D.F.; Davis, J.A.: The "late" anaemia of hamolytic disease of the newborn. Brit. J. Haemat, $1 \mathrm{~L}: 247,1965$

4 Robertson, SE.J.: Study of the anaemia following exchange transfusion in haemolytic diseasc of the newborn M.J. Australia 2: 250, 1956

5 Allen, F.H.: Diamond, L.K.: Erythroblastosis jetalis. III. N. Engl. J. Med. 257:761, 1957.

6 Diamond, L.K.: Erytroblastosis tetalis. Round table discussion. Pediatrics 10: 337, 1952.

7 Gairdner, D.: Marks, J.; Roscoe, J.D.: Blood formation in infancy. Part 11 . Normal erytropoiesis. Arch. Dis. Child. 27: 214,1952 .

8 Matoth, $Y_{\text {; }}$ Zaizov, R,; Vorsano, I.: Postnatal changes in some red cells parameters. Acta Paediat. Scand. 60: 317,1971 .

- Dallman, P.R. Anemia of prematurity. Ann. Rev. Med. 32: 143, 198I.

10 Kanto, W.P.; Marino, B.; Godwin, A.S.; Bunyapen, Ch. $A B O$ hemolitic disease: a compararive study of clinical severify and delayed anemia. Pediatrics 62: 365, 1978 .

11 Giblett, E.R.; Varela, J.E.: Finch, C.A.: Damage of the bone marnow due to $\mathbf{R h}$ antibody. Pediatrics 1: 37,1956 .

12 Dillon, H.C.: Krivit, W.: Serial study of bone marrow in hemolytic disease of the newborn (crythroblastosis fetalis). Pediatrics. 23: 314, 1959. 